\title{
ASYMPTOTICALLY STABLE MULTI-VALUED MANY-TO-MANY ASSOCIATIVE MEMORY NEURAL NETWORK AND ITS APPLICATION IN IMAGE RETRIEVAL
}

\author{
Lei Chen ${ }^{* \dagger}$, Geng Yang ${ }^{* \dagger}$ Yingzhou Zhang*, Chuandong Wang*, Zhen Yang ${ }^{\dagger}$
}

\begin{abstract}
As an important artificial neural network, associative memory model can be employed to mimic human thinking and machine intelligence. In this paper, first, a multi-valued many-to-many Gaussian associative memory model $\left(\mathrm{M}^{3} \mathrm{GAM}\right)$ is proposed by introducing the Gaussian unidirectional associative memory model (GUAM) and Gaussian bidirectional associative memory model (GBAM) into Hattori et al's multi-module associative memory model $\left((\mathrm{MMA})^{2}\right)$. Second, the $\mathrm{M}^{3}$ GAM's asymptotical stability is proved theoretically in both synchronous and asynchronous update modes, which ensures that the stored patterns become the $\mathrm{M}^{3}$ GAM's stable points. Third, by substituting the general similarity metric for the negative squared Euclidean distance in $\mathrm{M}^{3} \mathrm{GAM}$, the generalized multivalued many-to-many Gaussian associative memory model $\left(\mathrm{GM}^{3} \mathrm{GAM}\right)$ is presented, which makes the $\mathrm{M}^{3} \mathrm{GAM}$ become its special case. Finally, we investigate the $\mathrm{M}^{3}$ GAM's application in association-based image retrieval, and the computer simulation results verify the $\mathrm{M}^{3} \mathrm{GAM}$ 's robust performance.
\end{abstract}

Key words: Artificial neural network, associative memory model, asymptotical stability, similarity metric, association-based image retrieval

Received: September 16, 2011

Revised and accepted: March 20, 2013

\section{Introduction}

Associative memory model (AM) is an important artificial neural network which can be employed to mimic human thinking and machine intelligence [1-2]. In the

${ }^{*}$ Lei Chen, Geng Yang, Yingzhou Zhang, Chuandong Wang

School of Computer, Nanjing University of Posts and Telecommunications, E-mail: chenlei@njupt.edu.cn (L. Chen), yangg@njupt.edu.cn (G. Yang)

${ }^{\dagger}$ Lei Chen, Geng Yang, Zhen Yang

Key Laboratory of Broadband Wireless Communication \& Sensor Network Technology, Ministry of Education 
past decades, researchers have proposed many AMs such as the unidirectional associative memory model (UAM), bidirectional associative memory model (BAM) and many-to-many associative memory model $\left(\mathrm{M}^{2} \mathrm{AM}\right)$. Among all the existing AMs, Hopfield associative memory model (HAM) [3] is the most popular, which has yielded a great impact on the development of neural networks. However, HAM only addresses unidirectional association. The original bidirectional associative memory (BAM) is a two-layer neural network introduced by Kosko [4-5] as a variant of HAM. Kosko's BAM extends unidirectional auto-association to the bidirectional hetero-association. Owing to adoption of the Hebbian correlation learning scheme [6], the HAM and Kosko's BAM suffer from extremely low storage capacity and error-correcting capability. In addition, since Kosko's BAM only deals with two input/output patterns, Humpert tried to extend it to more input/output patterns and constructed the bidirectional associative memory with several patterns named $\operatorname{BAM}_{q}[7]$. The storage capability of the $\mathrm{BAM}_{q}$ can be improved significantly by adopting outer-product schemes or high order connection schemes, but it only addresses association with binary patterns. Therefore, some researchers continue to explore new architectures or learning schemes to enlarge the processed data range from just binary to multi-value, and simultaneously improve the AMs' performance. Among these works, Dembo and Zeitouni proposed the Potential-function correlation associative memory model (PCAM) [8]. Although the PCAM's capacity grows exponentially with the number of neurons, the primary disadvantage of this model is that hardware implementation can be cumbersome. Later, Chieuh and Goodman [9] employ the exponential correlation learning scheme and proposed the exponential correlation associative memory model (ECAM) with comparable storage capacity to PCAM which has an advantage of easier hardware implementation. Chiueh also proposed the multi-valued exponential correlation associative memory model (MV-ECAM) [10] by utilizing the same exponential correlation learning scheme to enlarge the correlation between stored pattern pairs. Furthermore, based on the concept of exponential correlation learning, Wang et al. proposed the multi-valued exponential bidirectional associative memory model (MV-eBAM) and investigated the impressive storage capacity and error-correcting capability of the MV-ECAM and MV-eBAM theoretically and experimentally [11].

The UAM and BAM deal with only one-to-one associations. In contrast, human memory can recall several related patterns satisfied with two or more query conditions simultaneously. For example, we can deal with many-to-many association such as an apple and cherry are recalled by red and fruit. The multi-module associative memory model $\left((\mathrm{MMA})^{2}\right)$ proposed by Hattori and Hagiwara [12-13] is the first successful neural network model which can realize many-to-many association. The (MMA) ${ }^{2}$ adopts the modular architecture. In each module, the (MMA) ${ }^{2}$ employs the modified HAM and BAM with the intersection learning algorithm to reconstruct the correct pattern from noisy or incomplete input. Nevertheless, the $(\mathrm{MMA})^{2}$ has two critical shortcomings as follows: (1) (MMA) ${ }^{2}$ can deal with only binary patterns; (2) (MMA) ${ }^{2}$ cannot store the training patterns online, that is, when a training pattern is stored, the weight matrix of (MMA) ${ }^{2}$ must be recalculated.

In this work, in order to address these shortcomings, we first propose the multivalued many-to-many Gaussian associative memory model $\left(\mathrm{M}^{3} \mathrm{GAM}\right)$ by introduc- 
ing the Gaussian unidirectional associative memory model (GUAM) and Gaussian bidirectional associative memory model (GBAM) into Hattori et al.'s (MMA) ${ }^{2}$. Then we prove that the $\mathrm{M}^{3} \mathrm{GAM}$ is asymptotically stable in both synchronous (all neurons in the output layer update themselves at the same time) and asynchronous (only one neuron in the output layer updates itself at a time) update modes by designing a bounded energy function, which can ensure that the stored patterns can be properly recalled theoretically. Further, by introducing the general similarity metrics, we propose the generalized multi-valued many-to-many Gaussian associative memory model $\left(\mathrm{GM}^{3} \mathrm{GAM}\right)$, which makes the $\mathrm{M}^{3} \mathrm{GAM}$ become its special case. Finally, we present an $\mathrm{M}^{3}$ GAM's application case for association-based image retrieval, and the computer simulation results verify the $\mathrm{M}^{3} \mathrm{GAM}$ 's robust performance.

The rest of this paper is organized as follows. Section 2 describes in detail the structure of $\mathrm{M}^{3} \mathrm{GAM}$, and analyzes its asymptotical stability theoretically. Section 3 proposes the generalized multi-valued many-to-many Gaussian associative memory model based on general similarity metric. Section 4 investigates the $\mathrm{M}^{3} \mathrm{GAM}$ 's application in association-based image retrieval and analyzes the $\mathrm{M}^{3}$ GAM's storage capacity and error-correcting capability. Finally, conclusions are given in Section 5 .

\section{Asymptotically Stable Multi-Valued Many-to-Many Gaussian Associative Memory Neural Network}

In this section, we first describe the structure of $\mathrm{M}^{3} \mathrm{GAM}$. Then, we discuss its stability or convergence in both synchronous and asynchronous update modes.

\subsection{Multi-valued many-to-many Gaussian associative memory model ( $\left.\mathrm{M}^{3} \mathrm{GAM}\right)$}

In order to describe the $\mathrm{M}^{3} \mathrm{GAM}$, we first introduce two basic components: Gaussian unidirectional associative memory model (GUAM) and Gaussian bidirectional associative memory model (GBAM).

Definition 1. Gaussian unidirectional associative memory model (GUAM) Suppose $\left\{X^{i} \mid i=1,2, \cdots, M\right\}$ are the stored patterns, $X=\left(x_{1}, x_{2}, \ldots, x_{n}\right)^{\mathrm{T}}$ is a current-state pattern to the network, and $X^{\prime}=\left(x_{1}^{\prime}, x_{2}^{\prime}, \ldots, x_{n}^{\prime}\right)^{\mathrm{T}}$ denotes its next recall state of $X$ in a dynamic update mode, where $X^{i}, X, X^{\prime} \in\{1,2, \ldots, L\}^{n}, L$ is the number of finite levels. If the UAM updates its state according to the following Gaussian exponential correlation learning rule:

$$
x_{k}^{\prime}=H\left(\frac{\sum_{i=1}^{M} x_{k}^{i} \cdot b^{-\left\|X^{i}-X\right\|^{2} / \sigma^{2}}}{\sum_{i=1}^{M} b^{-\left\|X^{i}-X\right\|^{2} / \sigma^{2}}}\right),
$$

where $b>1, \sigma \neq 0,-\left\|X^{i}-X\right\|^{2}$ denotes the negative squared Euclidean distance used to evaluate the similarity between the stored pattern $X^{i}$ and the input pattern 
$X, H(\cdot)$ is a staircase function shown as the following equation:

$$
H\left(u_{k}\right)=\left\{\begin{array}{l}
1, u_{k}<1 \\
L, u_{k}>L \\
\left\lfloor u_{k}+0.5\right\rfloor, u_{k} \in[1, L]
\end{array} .\right.
$$

Then, we call this one Gaussian unidirectional associative memory model (GUAM). Fig. 1 shows the structure of GUAM.

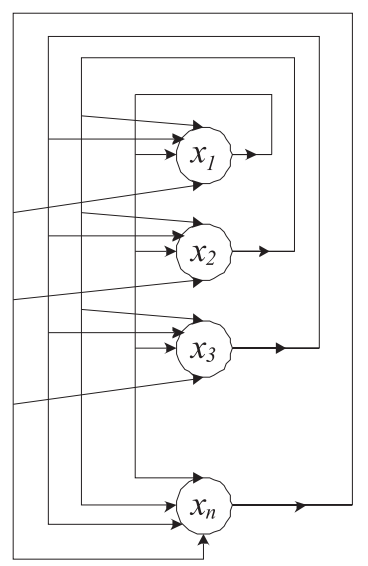

Fig. 1 Structure of the GUAM.

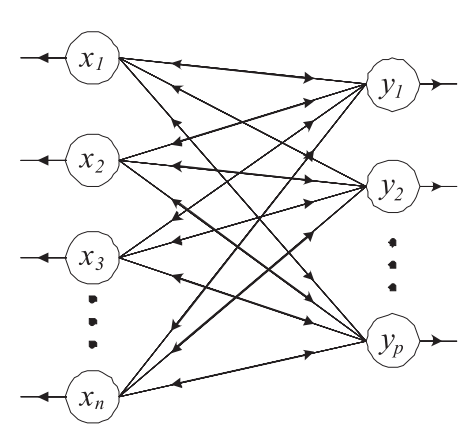

Layer-X

Fig. 2 Structure of the GBAM.

Definition 2 Gaussian bidirectional associative memory model (GBAM) Suppose $\left\{\left(X^{i}, Y^{i}\right) \mid i=1,2, \ldots, M\right\}$ are the stored pattern pairs, $X=\left(x_{1}, x_{2}, \ldots\right.$, $\left.x_{n}\right)^{\mathrm{T}}$ or $Y=\left(y_{1}, y_{2}, \ldots, y_{p}\right)^{\mathrm{T}}$ is a current-state pattern to the network, $X^{\prime}=$ $\left(x_{1}^{\prime}, x_{2}^{\prime}, \ldots, x_{n}^{\prime}\right)^{\mathrm{T}}$ and $Y^{\prime}=\left(y_{1}^{\prime}, y_{2}^{\prime}, \ldots, y_{p}^{\prime}\right)^{\mathrm{T}}$ denotes their next recall states in a dynamic update mode, where $X^{i}, X, X^{\prime} \in\{1,2, \ldots, L\}^{n}, Y^{i}, Y, Y^{\prime} \in\{1,2, \ldots, L\}^{p}$. If the BAM updates its state according to the following Gaussian exponential correlation learning rule:

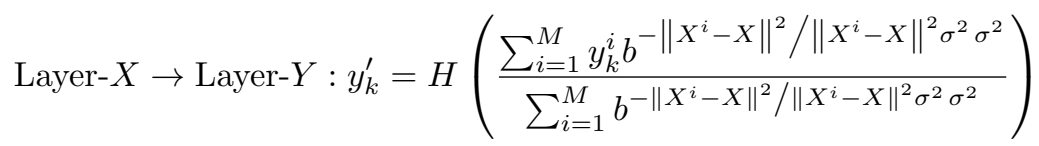

$$
\begin{aligned}
& \text { Layer- } Y \rightarrow \text { Layer- } X: x_{k}^{\prime}=H\left(\frac{\sum_{i=1}^{M} x_{k}^{i} b^{-\left\|Y^{i}-Y\right\|^{2} /\left\|Y^{i}-Y\right\|^{2} \sigma^{2} \sigma^{2}}}{\sum_{i=1}^{M} b^{-\left\|Y^{i}-Y\right\|^{2} /\left\|Y^{i}-Y\right\|^{2} \sigma^{2} \sigma^{2}}}\right),
\end{aligned}
$$

where $b>1, \sigma \neq 0, H(\cdot)$ is the same function as Equation (2). Then, we call this one Gaussian bidirectional associative memory model (GBAM). Fig. 2 shows the structure of GBAM. 
In order to construct a viable associative memory model for many-to-many association, Hattori and Hagiwara introduce the modular structure and the pseudonoise code, locally-represented pattern and logical operation into the (MMA) ${ }^{2}$ [12-13]. The (MMA) ${ }^{2}$ 's main strategies include: (1) each training sample must be numbered in advance; (2) each element of the training samples should be transformed into a locally-represented pattern; (3) logical operation between the locallyrepresented patterns is calculated; (4) each element of the training samples is memorized with the fundamental pseudo-noise code; (5) each training sample is memorized with the shifted pseudo-noise code.

The proposed $\mathrm{M}^{3} \mathrm{GAM}$ follows the same modular structure and association strategies as that in the (MMA) ${ }^{2}$. The main differences between the $\mathrm{M}^{3} \mathrm{GAM}$ and $(\mathrm{MMA})^{2}$ are the learning algorithm and the processed data range. The $\mathrm{M}^{3} \mathrm{GAM}$ employs the Gaussian exponential correlation learning algorithm instead of Hattori et al.'s intersection learning algorithm. More importantly, the $\mathrm{M}^{3}$ GAM can realize many-to-many association with multi-value patterns, which greatly expand the (MMA) ${ }^{2}$ 's practical application domains.

The $\mathrm{M}^{3} \mathrm{GAM}$ consists of four layers: Pseudo-Noise Pattern (PNP) layer, Input/Output (I/O) layer, Pre-Shift-Control (PSC) layer and Shift-Control (SC) layer. Fig. 3 shows the structure of $\mathrm{M}^{3} \mathrm{GAM}$. Note that the number of modules in the $\mathrm{M}^{3} \mathrm{GAM}$ is arbitrary, which depends on the processed data. In other words, if the processed data are $n$-tuple patterns, the number of modules must be n. As shown in Fig. 3, all the neurons of each module constitute a GBAM which is employed to realize the transformation between the element of the $n$-tuple training samples and the locally-represented patterns. At the same time, the neurons of the I/O layer in each module constitute a GUAM which is used to suppress the noise and reconstruct the corrected pattern when the noisy or incomplete query pattern is inputted to the I/O layer. In addition, the neurons of the I/O layer in each module and the neurons of the PNP layer constitute the GBAM to realize the storage between the element of the $n$-tuple training sample and the fundamental pseudo-noise code. The SC layer and the PSC layer in each module have the same

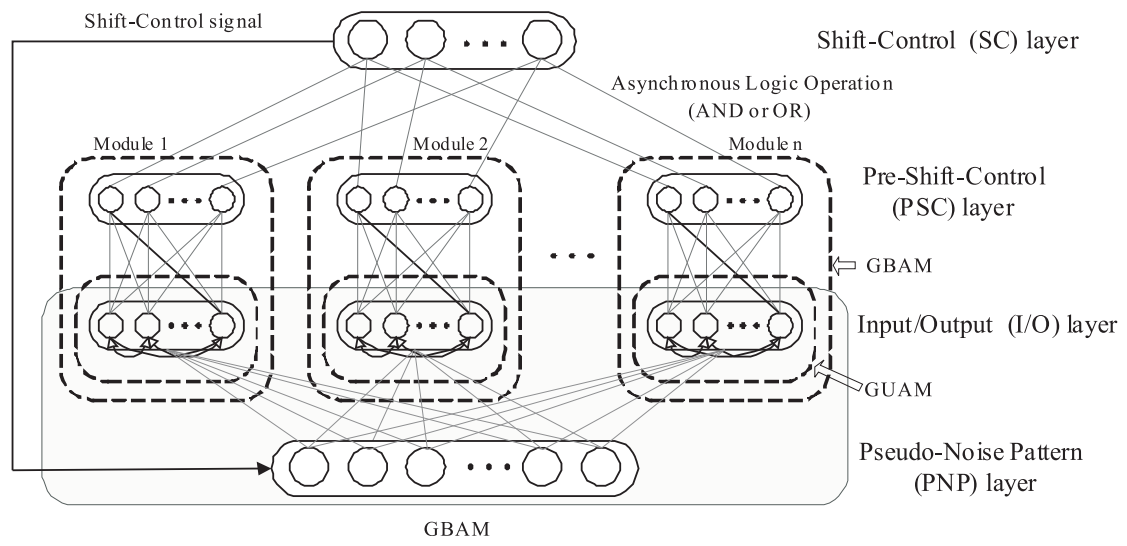

Fig. 3 Structure of the $M^{3}$ GAM. 
number of neurons, and the neurons in the PSC layer are connected to the corresponding neurons in the SC layer. Fig. 4 shows the connection between the PSC layer and the SC layer.

The $\mathrm{M}^{3}$ GAM's implementation procedure for many-to-many association will be described in detail in Section 4.

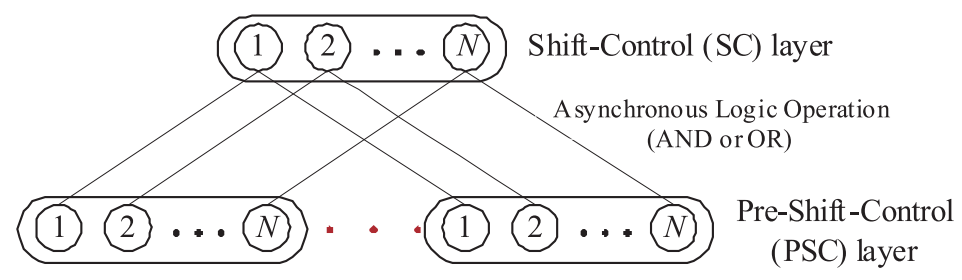

Fig. 4 Connection between the PSC layer and the SC layer.

\subsection{Asymptotical stability analysis of the $\mathrm{M}^{3} \mathrm{GAM}$}

Asymptotical stability is an important property of the associative memory neural network, which means that the network will converge to a fixed point, hopefully a stored prototype, from the initial input being the stored pattern with error. In this section, we intend to prove the $\mathrm{M}^{3} \mathrm{GAM}$ is asymptotically stable in both synchronous and asynchronous update modes.

Lemma 1 For any $m, n \in R$, if $b>1$, then $b^{m}-b^{n} \geq(\ln b) \cdot b^{n} \cdot(m-n)$.

Proof Let $f(x)=b^{x}$. Obviously, if $m=n$, then $b^{m}-b^{n}=(\ln b) \cdot b^{n} \cdot(m-n)=0$. Otherwise, because $f(x)$ is a continuous on the closed interval $[\min (m, n), \max (m, n)]$, and differentiable on the open interval $(\min (m, n), \max (m, n))$. According to Lagrange's mean value theorem, there exists some $\xi$ lying between $\min (m, n)$ and $\max (m, n)$ such that

$$
b^{m}-b^{n}=(\ln b) \cdot b^{\xi} \cdot(m-n) .
$$

Case 1: If $m>n$, then $m-n>0$ and $b^{\xi}>b^{n}$, thus $b^{m}-b^{n}>(m-n) \cdot \ln b \cdot b^{n}$.

Case 2: If $m<n$, then $m-n<0$ and $b^{\xi}<b^{n}$, thus $b^{m}-b^{n}>(m-n) \cdot \ln b \cdot b^{n}$.

Therefore, for any $m, n \in R$, if $b>1$, then $b^{m}-b^{n} \geq(\ln b) \cdot b^{n} \cdot(m-n)$.

Theorem 1 The GUAM is asymptotically stable in both synchronous and asynchronous update modes,

Proof In order to investigate GUAM's asymptotical stability, we only need to design a bounded energy function of GUAM, and then prove that the energy function will increase as the state of GUAM neural network changes. 
Lei Chen et al.: Asymptotically stable multi-valued many-to-many associative...

Now, let the energy function be defined as:

$$
E(X)=\sum_{i=1}^{M} b^{-\left\|X^{i}-X\right\|^{2} / \sigma^{2}}, b>1, \sigma \neq 0
$$

Obviously, $0<E(X) \leq M$, i.e., the energy function $E(X)$ is bounded.

Suppose $X^{\prime}$ is the next state of $X$, then the change of $E(X)$ is

$$
\begin{aligned}
\Delta E=E\left(X^{\prime}\right)-E(X) & =\sum_{i=1}^{M} b^{-\left\|X^{i}-X^{\prime}\right\|^{2} / \sigma^{2}}-\sum_{i=1}^{M} b^{-\left\|X^{i}-X\right\|^{2} / \sigma^{2}}= \\
& =\sum_{i=1}^{M}\left(b^{-\left\|X^{i}-X^{\prime}\right\|^{2} / \sigma^{2}}-b^{-\left\|X^{i}-X\right\|^{2} / \sigma^{2}}\right)
\end{aligned}
$$

According to Lemma 1, we have

$$
\begin{aligned}
& \Delta E \geq \sum_{i=1}^{M}\left[(\ln b) \cdot b^{-\left\|X^{i}-X\right\|^{2} / \sigma^{2}} \cdot\left(\left\|X^{i}-X\right\|^{2} / \sigma^{2}-\left\|X^{i}-X^{\prime}\right\|^{2} / \sigma^{2}\right)\right] \\
&=(\ln b) / \sigma^{2} \sum_{i=1}^{M}\left[b^{-\left\|X^{i}-X\right\|^{2} / \sigma^{2}} \cdot\left(2 X^{i}-X-X^{\prime}\right)^{\mathrm{T}}\left(X^{\prime}-X\right)\right] \\
&=(\ln b) / \sigma^{2} \sum_{i=1}^{M}\left[b^{-\left\|X^{i}-X\right\|^{2} / \sigma^{2}} \cdot \sum_{k=1}^{n}\left(2 x_{k}^{i}-x_{k}-x_{k}^{\prime}\right) \cdot\left(x_{k}^{\prime}-x_{k}\right)\right] \\
&=(\ln b) / \sigma^{2} \sum_{k=1}^{n}\left\{\sum_{i=1}^{M} b^{-\left\|X^{i}-X\right\|^{2} / \sigma^{2}} .\right. \\
&\left.\cdot\left[\left(2 \sum_{i=1}^{M} x_{k}^{i} \cdot b^{-\left\|X^{i}-X\right\|^{2} / \sigma^{2}} / \sum_{i=1}^{M} b^{-\left\|X^{i}-X\right\|^{2} / \sigma^{2}}-x_{k}-x_{k}^{\prime}\right) \cdot\left(x_{k}^{\prime}-x_{k}\right)\right]\right\} \\
& \text { Let } u_{k}=\sum_{i=1}^{M} x_{k}^{i} \cdot b^{-\left\|X^{i}-X\right\|^{2} / \sigma^{2}} / \sum_{i=1}^{M} b^{-\left\|X^{i}-X\right\|^{2} / \sigma^{2}}, \text { then } \\
& \qquad E \geq \\
&\qquad \ln b) / \sigma^{2} \cdot \sum_{i=1}^{M} b^{-\left\|X^{i}-X\right\|^{2} / \sigma^{2}} \cdot \sum_{k=1}^{n}\left\{\left[\left(u_{k}-x_{k}\right)+\left(u_{k}-x_{k}^{\prime}\right)\right] \cdot\left(x_{k}^{\prime}-x_{k}\right)\right\} \\
& \text { Let } s_{k}=\left[\left(u_{k}-x_{k}\right)+\left(u_{k}-x_{k}^{\prime}\right)\right] \cdot\left(x_{k}^{\prime}-x_{k}\right) . \\
& \text { According to Equations }(1) \text { and }(2), \text { we have the following different cases: }
\end{aligned}
$$

Case 1: If $u_{k}<1$, then $x_{k}^{\prime}=1, x_{k} \geq 1$, thus $s_{k} \geq 0$, and $s_{k}=0$ iff $x_{k}^{\prime}=x_{k}=1$.

Case 2: If $u_{k}>L$, then $x_{k}^{\prime}=L, x_{k} \leq L$, thus $s_{k} \geq 0$, and $s_{k}=0$ iff $x_{k}^{\prime}=x_{k}=L$.

Case 3: If $1 \leq u_{k} \leq L$, we have

(1) If $-0.5 \leq u_{k}-x_{k}<0.5$, then $x_{k}^{\prime}=\left\lfloor u_{k}+0.5\right\rfloor=x_{k}$, thus $s_{k}=0$.

(2) If $0.5 \leq u_{k}-x_{k}$, then $x_{k}^{\prime}=\left\lfloor u_{k}+0.5\right\rfloor>x_{k},-0.5 \leq u_{k}-x_{k}^{\prime}$, thus $s_{k} \geq 0$, and $s_{k}=0$ iff $x_{k}^{\prime}=x_{k}+1$ and $u_{k}-x_{k}=0.5$.

(3) If $u_{k}-x_{k}<-0.5$, then $x_{k}^{\prime}=\left\lfloor u_{k}+0.5\right\rfloor<x_{k}, u_{k}-x_{k}^{\prime}<0.5$, thus $s_{k}>0$.

From the above deduction, we can observe that $\Delta E \geq 0$. Therefore, with the state of GUAM changes, the bounded energy function $E(X)$ will converge to a fixed point. These facts imply that the GUAM will eventually reach a stable state with maximal energy level, i.e., the GUAM is asymptotically stable in both synchronous and asynchronous update modes.

Theorem 2 The GBAM is asymptotically stable in both synchronous and asynchronous update modes. 
Proof The GBAM is one kind of bidirectional associative memory models (BAMs). Therefore, we can investigate its stability by studying its two phase of evolution, i.e., Layer- $X \rightarrow$ Layer- $Y$ and Layer- $Y \rightarrow$ Layer- $X$.

(I) Layer- $X \rightarrow$ Layer- $Y$ phase

We define an energy function similar to that of GUAM,

$$
E_{X \rightarrow Y}(X, Y)=\sum_{i=1}^{M}\left\|X-X^{i}\right\|^{2} \cdot b^{-\left\|Y-Y^{i}\right\|^{2} / \sigma^{2}} .
$$

Obviously, $0 \leq E_{X \rightarrow Y}(X, Y) \leq M n L^{2}$, i.e., the energy function $E_{X \rightarrow Y}(X, Y)$ is bounded.

Suppose $X^{\prime}$ is the next state of $X$, then the change of $E_{X \rightarrow Y}(X, Y)$ can be derived to be

$$
\begin{aligned}
& \Delta_{X} E_{X \rightarrow Y}(X, Y)=E_{X \rightarrow Y}\left(X^{\prime}, Y\right)-E_{X \rightarrow Y}(X, Y)= \\
= & \sum_{i=1}^{M}\left[\left(\left\|X^{\prime}-X^{i}\right\|^{2}-\left\|X-X^{i}\right\|^{2}\right) \cdot b^{-\left\|Y-Y^{i}\right\|^{2} / \sigma^{2}}\right]= \\
= & \sum_{i=1}^{M}\left[\left(X^{\prime}+X-2 X^{i}\right)^{T}\left(X^{\prime}-X\right) \cdot b^{-\left\|Y-Y^{i}\right\|^{2} / \sigma^{2}}\right]= \\
= & \sum_{i=1}^{M} b^{-\left\|Y-Y^{i}\right\|^{2} / \sigma^{2}} \cdot \sum_{k=1}^{n}\left[\left(x_{k}+x_{k}^{\prime}-2 \cdot \sum_{i=1}^{M} x_{k}^{i} \cdot b^{-\left\|Y-Y^{i}\right\|^{2} / \sigma^{2}} /\right.\right. \\
& \left.\left./ \sum_{i=1}^{M} b^{-\left\|Y-Y^{i}\right\|^{2} / \sigma^{2}}\right) \cdot\left(x_{k}^{\prime}-x_{k}\right)\right] .
\end{aligned}
$$

Let $v_{k}=\sum_{i=1}^{M} x_{k}^{i} \cdot b^{-\left\|Y-Y^{i}\right\|^{2} / \sigma^{2}} / \sum_{i=1}^{M} b^{-\left\|Y-Y^{i}\right\|^{2} / \sigma^{2}}$, then

$\Delta_{X} E_{X \rightarrow Y}(X, Y)=\sum_{i=1}^{M} b^{-\left\|Y-Y^{i}\right\|^{2} / \sigma^{2}} \cdot \sum_{k=1}^{n}\left\{\left[\left(x_{k}-v_{k}\right)+\left(x_{k}^{\prime}-v_{k}\right)\right] \cdot\left(x_{k}^{\prime}-x_{k}\right)\right\}$

Let $r_{k}=\left[\left(x_{k}-v_{k}\right)+\left(x_{k}^{\prime}-v_{k}\right)\right] \cdot\left(x_{k}^{\prime}-x_{k}\right)$.

According to Equations (2-4), we have the following different cases:

Case 1: If $v_{k}<1$, then $x_{k}^{\prime}=1, x_{k} \geq 1$, thus $r_{k} \leq 0$, and $r_{k}=0$ iff $x_{k}^{\prime}=x_{k}=1$.

Case 2: If $v_{k}>L$, then $x_{k}^{\prime}=L, x_{k} \leq L$, thus $r_{k} \leq 0$, and $r_{k}=0$ iff $x_{k}^{\prime}=x_{k}=L$.

Case 3: If $1 \leq v_{k} \leq L$, we have

(1) If $0.5 \geq x_{k}-v_{k}>-0.5$, then $x_{k}^{\prime}=\left\lfloor v_{k}+0.5\right\rfloor=x_{k}$, thus $r_{k}=0$.

(2) If $x_{k}-v_{k} \leq-0.5$, then $x_{k}^{\prime}=\left\lfloor v_{k}+0.5\right\rfloor \geq x_{k}+1, x_{k}^{\prime}-v_{k} \leq 0.5$, thus $r_{k} \leq 0$, and $r_{k}=0$ iff $x_{k}^{\prime}=x_{k}+1$ and $x_{k}-v_{k}=-0.5$.

(3) If $x_{k}-v_{k}>0.5$, then $x_{k}^{\prime}=\left\lfloor v_{k}+0.5\right\rfloor<x_{k}, x_{k}^{\prime}-v_{k}>-0.5$, thus $r_{k}<0$.

From the above deduction, we can see that $\Delta_{X} E_{X \rightarrow Y}(X, Y) \leq 0$. Note that the energy function $E_{X \rightarrow Y}(X, Y)$ is bounded. Therefore, the Layer- $X \rightarrow$ Layer- $Y$ phase of the GBAM is proved to be asymptotically stable. 
(II) Layer- $Y \rightarrow$ Layer- $X$ phase

By the similar deduction as shown in Layer- $X \rightarrow$ Layer- $Y$ phase, we can also prove that Layer- $Y \rightarrow$ Layer- $X$ phase is asymptotically stable. The only difference is the definition of the bounded energy function in this phase. The energy function of this phase is similar to Equation (6),

$$
E_{Y \rightarrow X}(X, Y)=\sum_{i=1}^{M}\left\|Y-Y^{i}\right\|^{2} \cdot b^{-\left\|X-X^{i}\right\|^{2} / \sigma^{2}} .
$$

Since the deduction procedure is the same as that of the Layer- $X \rightarrow$ Layer- $Y$ phase, we do not repeat the discussion.

In short, the Layer- $X \rightarrow$ Layer- $Y$ phase always reduces the $E_{X \rightarrow Y}(X, Y)$, while the Layer- $Y \rightarrow$ Layer- $X$ phase always reduces the $E_{Y \rightarrow X}(X, Y)$. The evolution will be terminated when both $E_{X \rightarrow Y}(X, Y)$ and $E_{Y \rightarrow X}(X, Y)$ reach their respective local minima at which the pattern pairs are stored. Therefore, we can draw a conclusion that the GBAM is asymptotically stable in both synchronous and asynchronous update modes.

Theorem 3 The $\mathrm{M}^{3}$ GAM is asymptotically stable in both synchronous and asynchronous update modes.

Proof The $\mathrm{M}^{3} \mathrm{GAM}$ adopts loosely coupled modular structure, which includes four layers: the SC layer, PSC layer, I/O layer and PNP layer. Note that the asynchronous logic operation between SC layer and PSC layer, and the signal transfer operation between SC layer and PNP layer do not belong to the iterative operation, the stability of which is self-evident. Therefore, the $\mathrm{M}^{3}$ GAM's asymptotical stability mainly depends on the GUAM and GBAM. That is, the $\mathrm{M}^{3} \mathrm{GAM}$ is asymptotically stable if and only if the GUAM and GBAM are both asymptotically stable. Since the GUAM and GBAM's asymptotical stability has been proved theoretically in Theorems $\mathbf{1}$ and $\mathbf{2}$, we can easily draw a conclusion that the $\mathrm{M}^{3} \mathrm{GAM}$ is also asymptotically stable in both synchronous and asynchronous update modes.

\section{Generalized Multi-Valued Many-to-Many Gaussian Associative Memory Model Based on General Similarity Metric $\left(\mathrm{GM}^{3} \mathrm{GAM}\right)$}

In many machine learning problems, the similarity metric has critical impact on the success of learning algorithms such as clustering, classification, information retrieval and pattern recognition, etc. An appropriate similarity metric used to faithfully model the underlying relationships between the processed data is usually needed for achieving a promising algorithm performance. The commonly used Euclidean distance metric, as shown in GUAM and GBAM, can deal only with the data with spherically symmetrical distribution. Therefore, the performance may not be satisfactory when the data relations cannot be accurately modeled by the Euclidean distance. In fact, there is a large variety of applications for which the Euclidean distance fails to reflect the data proximities. Our previous works [14-16] also show that the Euclidean distance is not appropriate to evaluate web 
services' similarity and human faces' similarity. More importantly, the Euclidean distance assumes that each feature of data is equally important and independent from others. This assumption may not be always valid in real applications, especially when dealing with high-dimensional or sparse data where some features may not be tightly related to the topic of interest. In contrast, an appropriate similarity metric should identify important features and discriminate relevant and irrelevant features. Thus, supplying such a similarity metric is highly problem-dependent and determines the success or failure of the learning algorithm [17].

The general similarity metric includes:

(1) Cosine similarity metric:

$$
S_{C O S I}\left(X^{i}, X\right)=\sum_{j=1}^{n}\left(x_{j}^{i} \cdot x_{j}\right) /\left(\sqrt{\sum_{j=1}^{n}\left(x_{j}^{i}\right)^{2}} \cdot \sqrt{\sum_{j=1}^{n}\left(x_{j}\right)^{2}}\right)
$$

(2) Inner-product similarity metric: $S_{I N P R}\left(X^{i}, X\right)=\sum_{j=1}^{n}\left(x_{j}^{i} \cdot x_{j}\right)$

(3) Minkowski similarity metric: $S_{M I N K}\left(X^{i}, X\right)=-\left(\sum_{j=1}^{n}\left|x_{j}^{i}-x_{j}\right|^{q}\right)^{1 / q}$

(4) Mahalanobis similarity metric:

$S_{M A H A}\left(X^{i}, X\right)=-\left(X^{i}-X\right)^{\mathrm{T}} * M^{-1} *\left(X^{i}-X\right)$, where $M^{-1}$ is the inverse matrix of pattern correlation matrix $M$.

(5) Canberra similarity metric:

$S_{C A N B}\left(X^{i}, X\right)=-\frac{1}{n} \sum_{j=1}^{n}\left|x_{j}^{i}-x_{j}\right| /\left(\left|x_{j}^{i}\right|+\left|x_{j}\right|\right)$

(6) Bray-Curtis similarity metric:

$$
S_{B R C U}\left(X^{i}, X\right)=-\sum_{j=1}^{n}\left|x_{j}^{i}-x_{j}\right| / \sum_{j=1}^{n}\left|x_{j}^{i}+x_{j}\right|
$$

(7) Pearson correlation coefficient similarity metric:

$$
S_{P E C O}\left(X^{i}, X\right)=\frac{\left(\sum_{j=1}^{n} x_{j}^{i} * x_{j}-\frac{1}{n} \sum_{j=1}^{n} x_{j}^{i} * \sum_{j=1}^{n} x_{j}\right)}{\left(\sqrt{\sum_{j=1}^{n}\left(x_{j}^{i}\right)^{2}-\frac{1}{n}\left(\sum_{j=1}^{n} x_{j}^{i}\right)^{2}} * \sqrt{\sum_{j=1}^{n}\left(x_{j}\right)^{2}-\frac{1}{n}\left(\sum_{j=1}^{n} x_{j}\right)^{2}}\right)}
$$

Based on the above considerations, we can naturally extend the $\mathrm{M}^{3} \mathrm{GAM}$ by substituting the general similarity metric for the negative squared Euclidean distance in the evolution rules of the GUAM and GBAM, and construct the generalized multi-valued many-to-many Gaussian associative memory model ( $\left.\mathrm{GM}^{3} \mathrm{GAM}\right)$. Suppose $S\left(X^{i}, X\right)$ denotes the general similarity metric between pattern $X^{i}$ and $X$, the evolution rules used in the $\mathrm{GM}^{3} \mathrm{GAM}$ can be described as follows:

(1) Evolution rule for the corresponding UAM:

$$
x_{k}^{\prime}=H\left(\frac{\sum_{i=1}^{M} x_{k}^{i} * b^{S\left(X^{i}, X\right) / \sigma^{2}}}{\sum_{i=1}^{M} b^{S\left(X^{i}, X\right) / \sigma^{2}}}\right)
$$


(2) Evolution rules for the corresponding BAM:

$$
\begin{gathered}
\text { Layer- } X \rightarrow \text { Layer- } Y: y_{k}^{\prime}=H\left(\frac{\sum_{i=1}^{M} y_{k}^{i} b^{-S\left(X^{i}, X\right) / \sigma^{2}}}{\sum_{i=1}^{M} b^{-S\left(X^{i}, X\right) / \sigma^{2}}}\right) \\
\text { Layer- } Y \rightarrow \text { Layer- } X: x_{k}^{\prime}=H\left(\frac{\sum_{i=1}^{M} x_{k}^{i} b^{S\left(Y^{i}, Y\right) / \sigma^{2}}}{\sum_{i=1}^{M} b^{S\left(Y^{i}, Y\right) / \sigma^{2}}}\right)
\end{gathered}
$$

Obviously, various general similarity metrics can be easily applicable to the proposed GM ${ }^{3} \mathrm{GAM}$. In particular, when $S\left(X^{i}, X\right)$ denotes the Minkowski similarity metric $\left(-\left(\sum_{j=1}^{n}\left|x_{j}^{i}-x_{j}\right|^{q}\right)^{1 / q}, q=2\right)$, the $\mathrm{M}^{3} \mathrm{GAM}$ becomes the $\mathrm{GM}^{3}$ GAM's special case.

\section{Application Case: Association-Based Image Retrieval}

Currently, text-based and content-based image retrieval are the two dominant image retrieval approaches [18]. In the text-based image retrieval (TBIR) systems, the images must be manually annotated by text descriptors. There are two main disadvantages with this approach [18-19]. The first is that a lot of human labor is required for manual annotation. The second is the annotation inaccuracy due to the subjectivity of human perception. In order to overcome the above disadvantages, content-based image retrieval (CBIR) was introduced [18-20]. In CBIR systems, images are indexed by their visual content, such as color, texture, shapes. However, CBIR systems depend on human interaction and the query results returned rely heavily on the similarity measure between query image and stored images. In contrast, the human memory can naturally associate several related images from some noisy or incomplete query images. Inspired by the idea of association, Kulkarni et al. first proposed a novel approach for image storage and retrieval named association-based image retrieval (ABIR) [21-22]. They use a generalized binary bidirectional associative memory model to store the relation between feature vectors that represent images stored in the database. Although Kulkarni's ABIR system is theoretically viable and shows the promising results on their test datasets, they still suffer from some disadvantages. For example, the Kulkarni's ABIR system is difficult to realize many-to-many association or conditional association. In other words, it only enables context association-based image retrieval, but cannot realize intersection association-based image retrieval and union association-based image retrieval. In this section, we will employ the proposed $\mathrm{M}^{3} \mathrm{GAM}$ to carry out the context/intersection/union association-based image retrieval.

As shown in Tab. I, there are nine 4-tuple training samples stored in the $\mathrm{M}^{3} \mathrm{GAM}$. Each training sample consists of four related images that represent faces, fruits, buildings and landscapes, respectively. The $\mathrm{M}^{3} \mathrm{GAM}$ includes 4 modules and module 1 stores faces, module 2 stores fruits, module 3 stores buildings, and module 4 stores landscapes. All the images are the 256 -level grayscale images with $60 \times 60$ 
pixels. In the data preprocessing stage, each image is turned into a 256-level vector by the row by row concatenation way. First, each 4-tuple training sample is numbered from 1 to 9 , respectively. Then, each element (image) of the 4-tuple training sample is transformed into a locally-represented pattern (as shown in Tab. II). For example, since the 'face_2' appears in the No. 2, No. 5, and No. 6 training sample, it is transformed into the locally-represented pattern (010011000). In this locallyrepresented pattern, the length of (010011000) indicates the number of the training samples, and 0 indicates the 'face_2' appears, while 1 indicates the 'face_2' does not appear. With the same way, we have the other locally-represented patterns, as shown in Tab. II. In order to carry out many-to-many association, all the training samples must be stored in the $\mathrm{M}^{3} \mathrm{GAM}$ with a set of shifted Pseudo-Noise $(P N)$ codes (the detailed explanation of pseudo-noise code can be found in Reference [23]). Let the fundamental $P N$ code be $P N_{0}$ for example: (100010011010111). Then, each element of the training sample is memorized with $P N_{0}$ in the GBAM between the I/O layer and the PNP layer so that $P N_{0}$ can be recalled by each element of the training sample. On the other hand, each training sample is memorized with the corresponding shifted $P N$ code in the GBAM between the I/O layer and the PNP layer so that training sample can be recalled by the corresponding shifted $P N$ code. For example, the No. 1 training sample is memorized with the shifted $P N$ code- $P N_{1}$ which is generated by shifting $P N_{0}$ with one bit. The No. 2 training sample is memorized with $P N_{2}$ which is generated by shifting $P N_{0}$ with two bits. With the same way, the No. 3 - No. 9 samples are memorized with $P N_{3}-P N_{9}$, respectively.

When the noisy or incomplete images are inputted to the I/O layer in the $M^{3}$ GAM, first the auto-association is carried out by the GUAM. After the stable state is reached, the corresponding locally-represented patterns and the fundamental pseudo-noise code are recalled by the GBAMs, respectively. As for the module which has no input, the states of all neurons in the PSC layer are set to 1 (context and intersection association) or 0 (union association) for the logical AND (context and intersection association) or OR (union association) operation in the SC layer. Then, the neurons in the SC layer calculate logical AND or logical OR asynchronously and compete to change their states. Suppose that the state of the SC layer is (000100000), the $P N_{0}$ is shifted with 4 bits. Thus, using the GBAM between the PNP layer and the I/O layer, the No. 4 training sample is recalled by the $\mathrm{PN}_{4}$ as one of the output results. Then, the winner neuron in the SC layer is inhibited afterward. Therefore, another desired output is recalled successively by the similar retrieval procedure.

In this simulation, since each image has $60 \times 60$ pixels, the I/O layer in each module consists of 3600 neurons. At the same time, the number of neurons in the PNP layer is 15, which equals to the number of bits of the $P N_{0^{-}}(100010011010111)$. Since only nine training samples are stored in the $\mathrm{M}^{3} \mathrm{GAM}$, the number of neurons in the PSC layer and the SC layer are 9 and 9 , respectively. In addition, we set the parameters of the GUAM and GBAM to be $b=2, \sigma=3$ due to their experimentally proven better performances. 
Lei Chen et al.: Asymptotically stable multi-valued many-to-many associative...

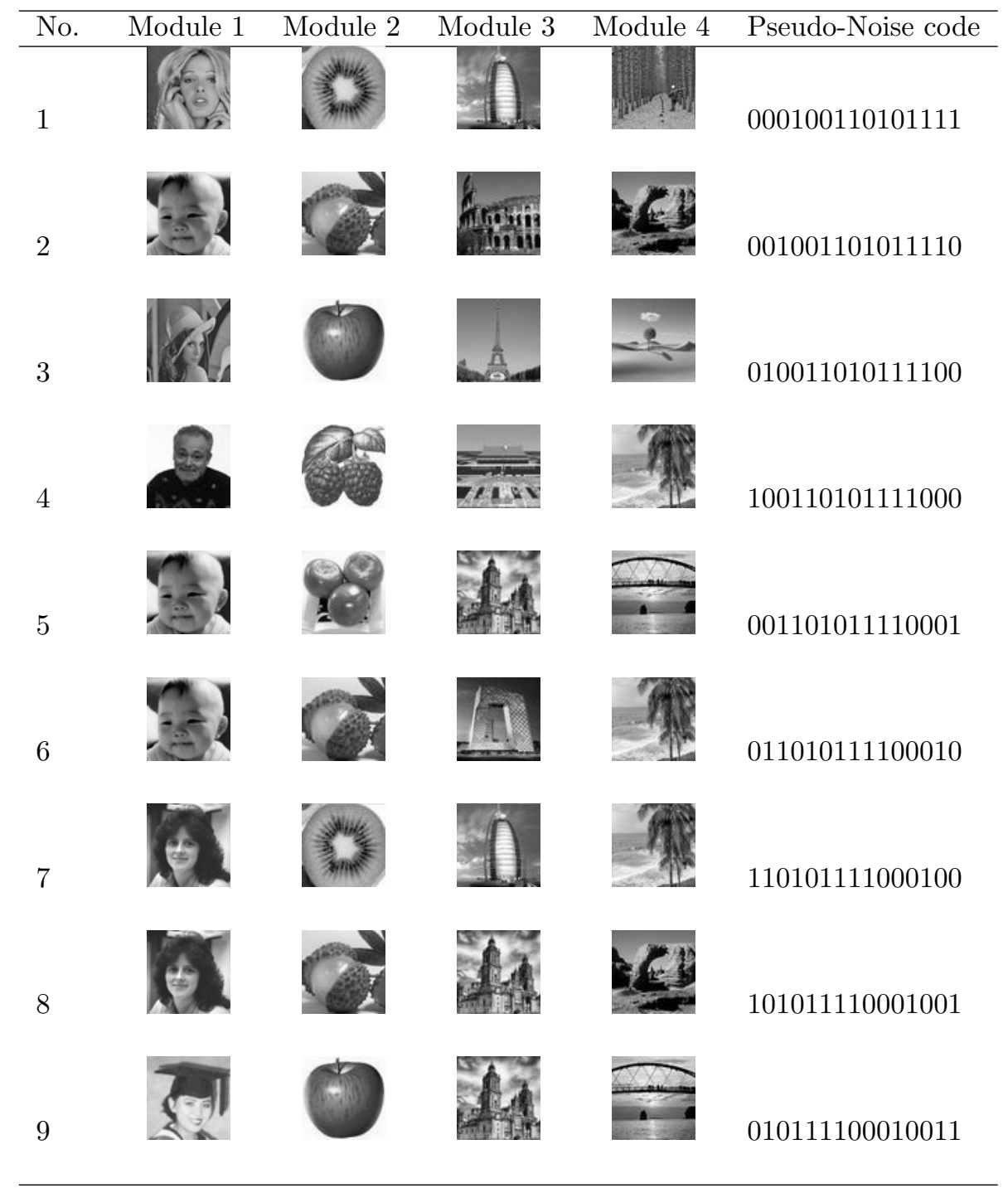

Tab. I The stored training samples and their Numbers and Pseudo-Noise codes.

\subsection{Context association-based image retrieval}

Context association is the basic function of the $\mathrm{M}^{3} \mathrm{GAM}$. When a single original image or its low-noisy one was inputted to the $\mathrm{M}^{3} \mathrm{GAM}$ as the query request, all the stored training samples containing this image will be recalled correctly. For example, suppose 9 training samples shown in Tab. I have been stored in the $\mathrm{M}^{3}$ GAM. Even if the testing image 'face_2' with $25 \%$ salt \& pepper noise is applied to the $\mathrm{M}^{3} \mathrm{GAM}$, we can clearly see from Fig. 5 that the $\mathrm{M}^{3} \mathrm{GAM}$ is still able to exactly retrieve the No. 2, No. 5, and No. 6 training sample. 
Neural Network World 2/13, 169-189

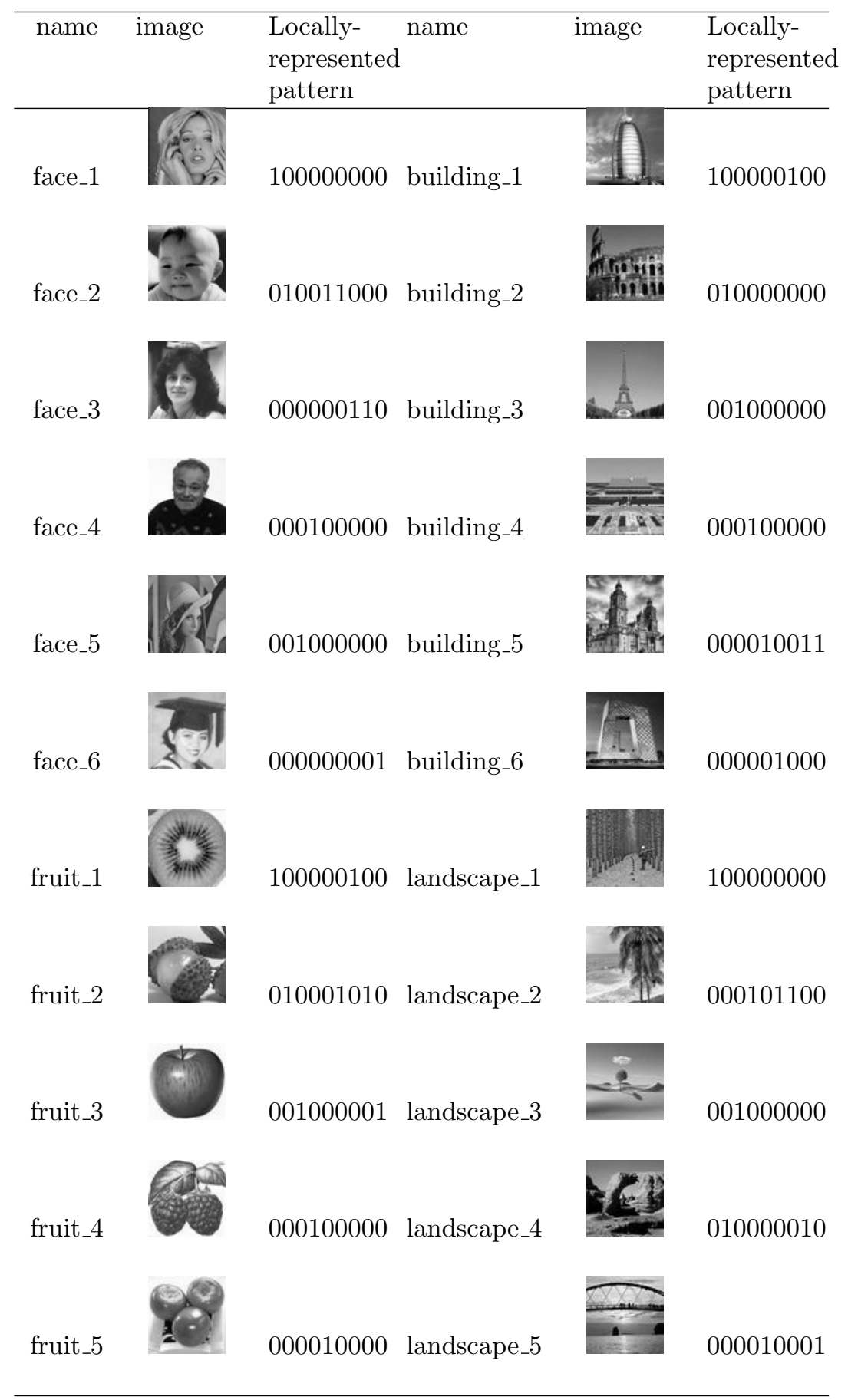

Tab. II The elements of training samples and the corresponding locally-represented pattern. 


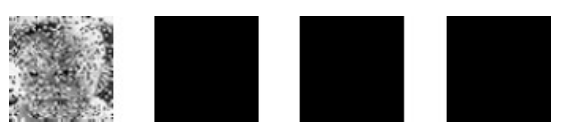

(a) The initial input image with $25 \%$ salt \& pepper noise

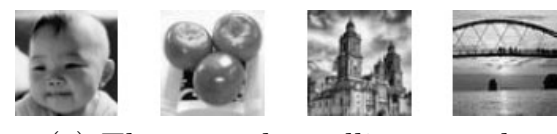

(c) The second recalling sample

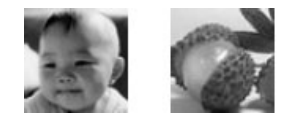

(b) The first recalling sample
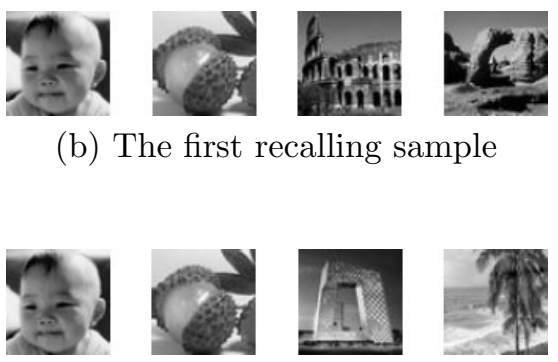

(d) The third recalling sample

Fig. 5 Context association-based image retrieval.

\subsection{Intersection association-based image retrieval}

When the logical AND operation is adopted in the SC layer, and the initial states of all neurons in the PSC layer are set to 1 , the $\mathrm{M}^{3} \mathrm{GAM}$ can carry out the intersection association-based image retrieval. If the multiple inputs are applied to the $\mathrm{M}^{3} \mathrm{GAM}$, the outputs to be recalled will be determined by the intersection of the data sets which include these inputs. Suppose the $\mathrm{M}^{3} \mathrm{GAM}$ stored the training samples as shown in Tab. I. Even if the testing images 'fruit_1' with $25 \%$ salt \& pepper noise and 'building_1' with Gaussian white noise (0 mean, 0.05 variance) are applied to the $\mathrm{M}^{3} \mathrm{GAM}$ as an intersection query request, we can clearly see from Fig. 6 that the $\mathrm{M}^{3} \mathrm{GAM}$ is still able to exactly retrieve the No. 1 and No. 7 training sample.
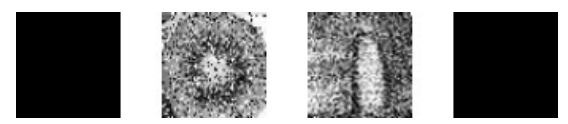

(a) The initial input images with $25 \%$ salt \& pepper noise and Gaussian white noise (0 mean, 0.05 variance)
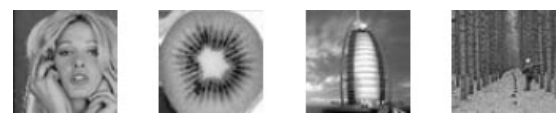

(b) The first recalling sample
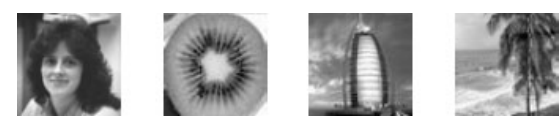

(c) The second recalling sample

Fig. 6 Intersection association-based image retrieval.

\subsection{Union association-based image retrieval}

In addition to the intersection association, the $\mathrm{M}^{3} \mathrm{GAM}$ can also deal with the union association by changing the logical AND operation into logical OR operation, and the initial states of all neurons in the PSC layer should be set to 0. For example, when the testing images 'fruit_3' with Gaussian white noise (0 mean, 0.04 variance) and 'landscape_4' with $25 \%$ occlusion are applied to the $\mathrm{M}^{3} \mathrm{GAM}$, the No. 3 , No. 4, No. 6, No. 7 and No. 9 training sample can be correctly retrieved. Fig. 7 shows the simulation results. 

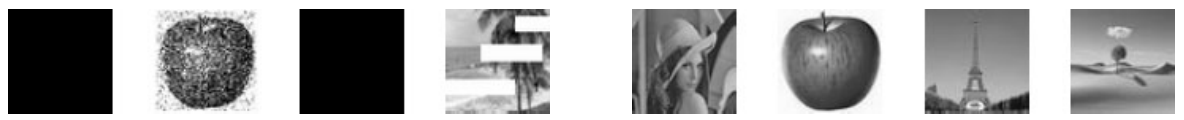

(a) The initial input images with

$25 \%$ occlusion and Gaussian white noise (0 mean, 0.04 variance)
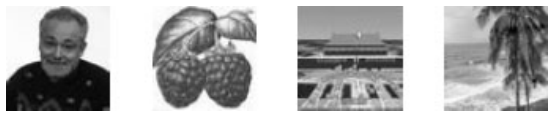

(c) The second recalling sample
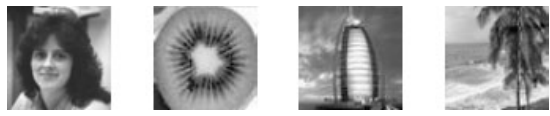

(e) The fourth recalling sample (b) The first recalling sample

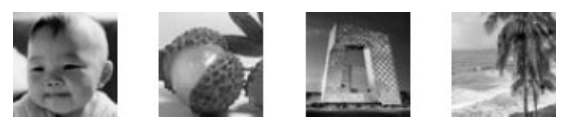

(d) The third recalling sample

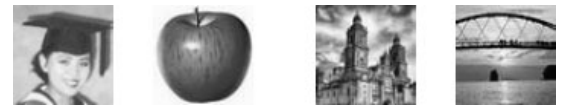

(f) The fifth recalling sample

Fig. 7 Union association-based image retrieval.

\subsection{Performance analysis of the $\mathrm{M}^{3} \mathrm{GAM}$}

Since the performance of the Associative Memory model is usually evaluated in terms of storage capacity and error-correction capability, in this subsection, we will first analyze the $\mathrm{M}^{3}$ GAM's storage capacity, and then investigate the $\mathrm{M}^{3}$ GAM's error-correction capability.

\subsubsection{Storage capacity analysis of the $\mathrm{M}^{3} \mathrm{GAM}$}

From the structure of the $\mathrm{M}^{3} \mathrm{GAM}$, we can clearly observe that its storage capacity not only depends on the GUAM and GBAM, but also depends on the numbers of neurons in each layer. On the one hand, previous works [9-11, 15-16] have demonstrated that exponential correlation learning schemes can lead to the Associative Memory model's large storage capacity. Note that the GUAM and GBAM adopt the Gaussian exponential correlation learning scheme which is the natural generalization of such schemes. In particular, if the parameter $\sigma$ is set to 1 and the staircase function $H(\cdot)$ is replaced with that given by Wang et al [11], the GUAM and GBAM will become the MV-ECAM and MV-eBAM respectively which have been proved theoretically and verified experimentally to possess a large storage capacity [11]. On the other hand, although the $\mathrm{M}^{3}$ GAM's storage capacity is restricted by the numbers of neurons in the SC layer, the PSC layer and the PNP layer, the storage capacity becomes large as the number of neurons in these layers becomes large. Usually, the number of neurons in the PSC layer should be greater than or equal to the number of patterns to be stored. While the number of neurons in the SC layer must be equal to that in the PSC layer. the number of neurons in the PNP layer must be equal to the length of the PN code selected. In addition, we can see that the $\mathrm{M}^{3} \mathrm{GAM}$ can store more patterns by using two or more fundamental $P N$ codes even if the number of the neurons in the PNP layer is unchanged. 
Since each $P N$ code has the same characteristics [23], the choice of the $P N$ codes does not affect the performance of the $\mathrm{M}^{3} \mathrm{GAM}$. Hence, we can easily improve the storage capacity of the $\mathrm{M}^{3} \mathrm{GAM}$ by increasing the number of neurons in the $\mathrm{SC}$ layer, the PSC layer and the PNP layer.

\subsubsection{Error-correction capability analysis of the $\mathrm{M}^{3} \mathrm{GAM}$}

Many researchers have demonstrated that Associative Memory models contain very strong robustness to noisy and incomplete inputs [9-11, 15-16]. Now we perform some experiments to show that $\mathrm{M}^{3} \mathrm{GAM}$ has a similar performance. Since the $\mathrm{M}^{3} \mathrm{GAM}$ employs the GUAM to suppress the noise and reconstruct the pattern when the noisy or incomplete query pattern is inputted, from Fig. 3 we can easily observe that the $\mathrm{M}^{3} \mathrm{GAM}$ 's error-correction capability mainly depends on the GUAM. Thus, in this subsection, we investigate the GUAM's error-correction capability by adding random noises such as Gaussian white noise and salt \& pepper noise to the grayscale images or occluding partly grayscale images with white block noise.

In this experiment, we gather the 600 256-level grayscale images with $60 \times 60$ pixels from public Search Engine - Google as our target database, which consists of faces, fruits, buildings, landscapes, animals, flowers, etc. Fig. 8(a) shows some sample images in this database. Figs. 8(b) and (c) show a set of testing images generated by adding the salt \& pepper noise or Gaussian white noise to the original training images, respectively. Fig. 8(d) shows the testing images corrupted with $6 \times 6$ white block noise. In the data preprocessing stage, each one of the 600 original images is first turned into a corresponding vector by the row by row concatenation way. When a noisy or incomplete image is inputted to the GUAM, if the output image matches entirely the original training exemplar, then we call a successful recognition. The high recognition rate means that the GUAM has a high errorcorrection capability.

Tabs. III-V show the average recognition rates of 10 thousand times independent repeated runs. From Tab. III, we can clearly observe that GUAM achieves as high as $100 \%$ recognition rate when salt \& pepper noise is not greater than $30 \%$. Meanwhile, Tab. IV also shows that GUAM has an impressive recognition rate even when the images are corrupted by Gaussian white noise with 0 mean and 0.3 variance. Note that at such Gaussian white noise level as shown in Figure 8(c), the recognition problem becomes extremely difficult even for the human. In addition, when $6 \times 6$ white block occlusion is not serious, for example, not greater than $15 \%$, as shown in Tab. V, GUAM obtains almost 100\% recognition rate. More importantly, from all the experiments, we can clearly see that the GUAM converges and gets stability after only less than three iterations on the average.

In addition, since the GBAM is employed to realize the transformation or recall between the grayscale images and the binary locally-represented patterns or pseudo-noise codes in the $\mathrm{M}^{3} \mathrm{GAM}$, we also carry out two preliminary experiments to investigate the GBAM's transformation/recall capability by constructing two sets of pattern pairs, respectively. In the first experiment, we randomly generate 600 different 30 -dimensional binary vectors, whose average hamming distance is 10.34. Each pattern pair consists of one binary vector and one grayscale im- 

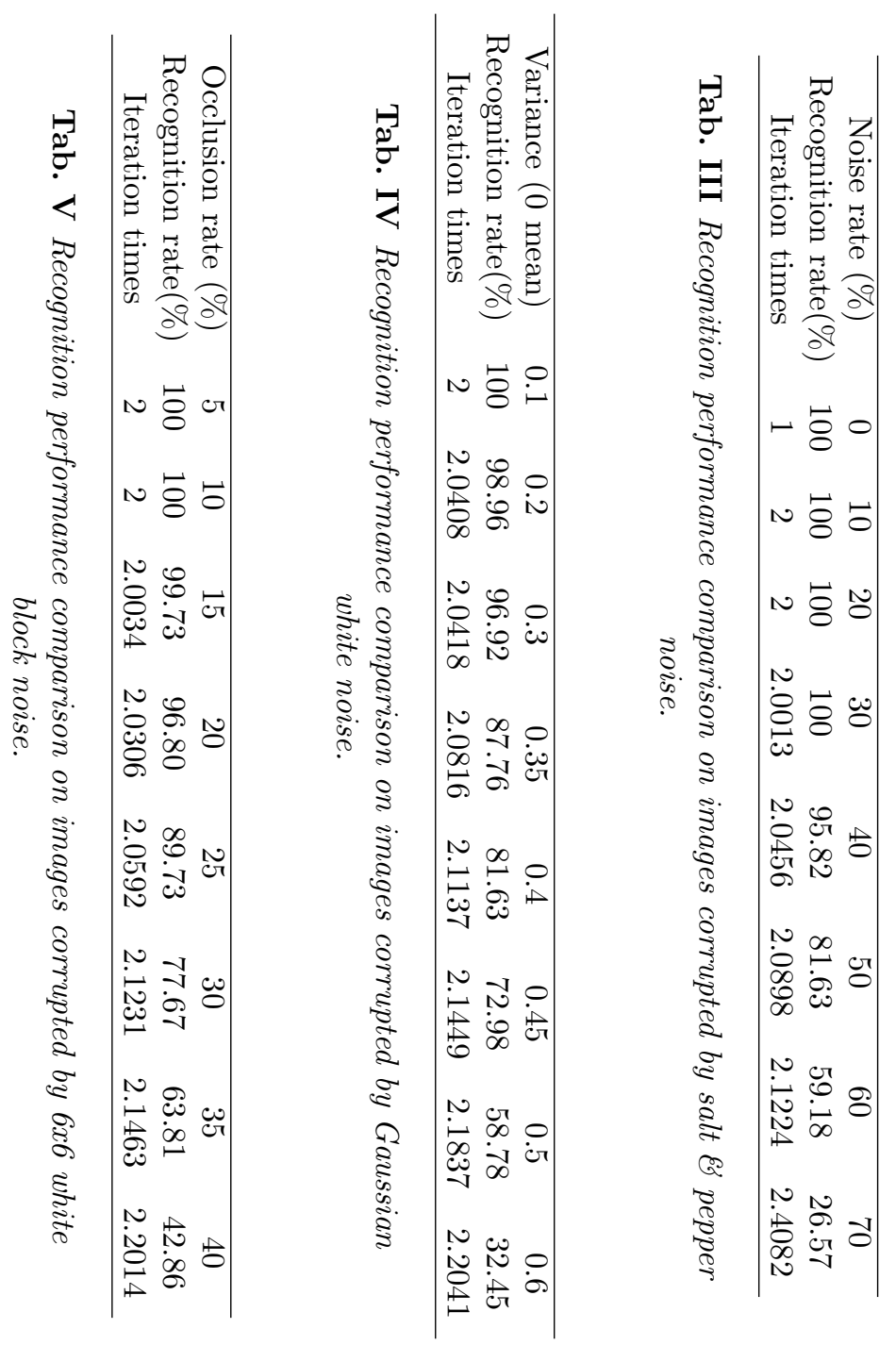


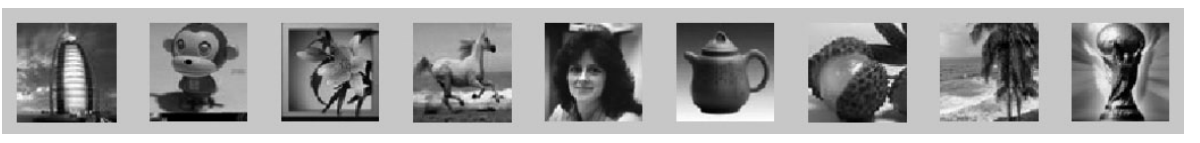

(a) Original training images

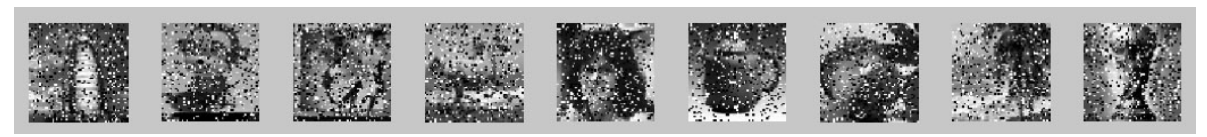

(b) testing images with $20 \%$ salt \& pepper noise

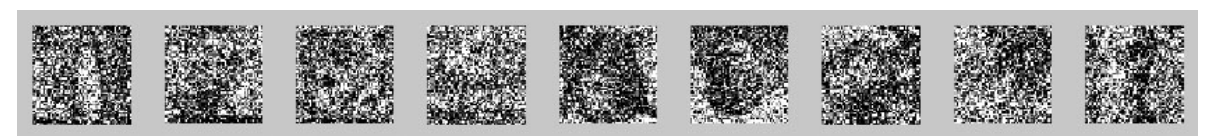

(c) Testing images with Gaussian white noise (0 mean, 0.3 variance)

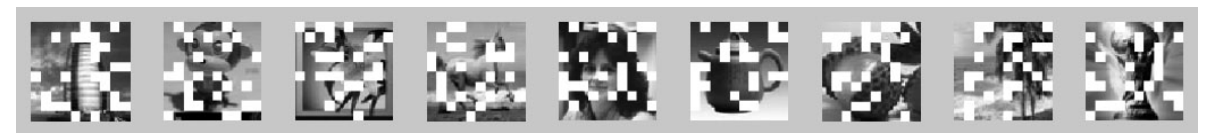

(d) Testing images with $25 \%$ occlusion (corrupted by $6 \times 6$ white block noise randomly)

Fig. 8 Examples of the training images and the testing images.

age from the above images database. Experimental result shows that the recall probability is up to $100 \%$. In the second experiment, we specially select another 600 different 30-dimensional binary vectors, whose average hamming distance is 4.18. In other words, for each one of these vectors, there must exist one "close" vector, and their hamming distance is less than or equal to 4 . Here each pattern pair consists of such one binary vector and one grayscale image from the same images database. Experimental result shows that the recall probability is as low as $21.52 \%$. Note that although the two experiments are performed on the same image database, their recall probabilities are obviously different, which may be because the correlation between the latter binary vectors is much higher than that between the former binary vectors. Therefore, from the two experiments, we seem able to infer indirectly that if the patterns are very "close" to each other, the $\mathrm{M}^{3} \mathrm{GAM}$ may produce incorrect recall results. Intuitively, this case is indeed consistent with our human recall mechanism.

\section{Conclusions and Future Work}

In this paper, we first propose the asymptotically stable multi-valued many-tomany Gaussian associative memory model $\left(\mathrm{M}^{3} \mathrm{GAM}\right)$ by introducing the GUAM and GBAM into Hattori et al.'s (MMA) ${ }^{2}$. Then, inspired by the idea of metric 
learning, we employ the general similarity metric to extend the $\mathrm{M}^{3} \mathrm{GAM}$, and construct the generalized multi-valued many-to-many Gaussian associative memory neural network $\left(\mathrm{GM}^{3} \mathrm{GAM}\right)$, which makes the $\mathrm{M}^{3} \mathrm{GAM}$ become its special case. Finally, we investigate one of the $\mathrm{M}^{3} \mathrm{GAM}$ 's application cases for association-based image retrieval, and the computer simulation results show that the $\mathrm{M}^{3} \mathrm{GAM}$ can not only exactly carry out many-to-many association, but also exhibit the robust error-correcting capability even to noisy and incomplete inputs.

In the future, we will investigate the characteristics and the behavior of the proposed $\mathrm{GM}^{3} \mathrm{GAM}$ experimentally and theoretically. In addition, we intend to improve the $\mathrm{M}^{3} \mathrm{GAM}$ and $\mathrm{GM}^{3} \mathrm{GAM}$ so that they can better mimic human thinking to deal with the more complex intelligent information processing problems.

\section{Acknowledgement}

The authors want to thank anonymous reviewers very much for their valuable comments and suggestions. This work was supported by the National Natural Science Foundation (Nos. 61272084, 61202004, 61202353), the National Key Basic Research Development Program (No. 2011CB302903), and the Natural Science Foundation of Jiangsu Province (No BK2011754), the Innovation Project for Postgraduate Cultivation of Jiangsu Province (No. CX10B_195Z), the Key University Natural Science Research Program of Jiangsu (No. 11KJA520002), the Research Fund for the Doctoral Program of High Education (No. 20113223110003 and 20093223120001).

\section{References}

[1] Haykin S.: Neural networks and learning machines, 3rd edition. Prentice Hall, Englewood Cliffs, NJ, 2009.

[2] Kosko B.: Neural Network and Fuzzy Systems. Englewood Cliffs, NJ: Prentice Hall, 1992.

[3] Hopfield J. J.: Neural network and physical systems with emergent collective computational abilities. Proceedings of the National Academy of Sciences, 1982, 79, 8, pp. 2554-2558.

[4] Kosko B.: Adaptive bidirectional associative memories. Applied Optics, 1987, pp. 353-359.

[5] Kosko B.: Bidirectional associative memories. IEEE Transactions on Systems, Man and Cybernetics, 1988, pp. 49-60.

[6] Hebb D. O.: The organization of behavior: a neuropsychological theory. Wiley, New York, 1949.

[7] Humpert B.: Bi-directional associative memory with several patterns. Proceedings of the International Joint Conference on Neural Networks, San Diego, 1990, pp. 741-750.

[8] Dembo A., Zeitouni O.: General potential surfaces and neural networks. Physics Review A, 1988, 37, 6, pp. 2134-2143.

[9] Chiueh T. D., Goodman R. M.: Recurrent correlation associative memories. IEEE Transactions on Neural Networks, 1991, 2, 2, pp. 275-284.

[10] Chiueh T. D., Tsai H. K.: Multivalued Associative memories based on recurrent networks. IEEE Transactions on Neural Networks, 1993, 4, 2, pp. 364-366.

[11] Wang C. C., Hwang S. M., Lee J. P.: Capacity analysis of the asymptotically stable multivalued exponential bidirectional associative memory. IEEE Transactions on Systems, Man, and Cybernetics, 1996, 26, 5, pp. 733-743.

[12] Hattori M., Hagiwara M.: Multimodule associative memory for many-to-many associations. Neurocomputing, 1998, 19, 1-3, pp. 99-119. 
Lei Chen et al.: Asymptotically stable multi-valued many-to-many associative...

[13] Hattori M., Hagiwara M.: Associative memory for intelligent control. Mathematics and Computers in Simulation, 2000, 51, 3-4, pp. 349-374.

[14] Chen L., Yang G., Wang D. R., Zhang Y. Z.: WordNet-powered web services discovery using kernel-based similarity matching mechanism. Fifth IEEE International Symposium on Service Oriented System Engineering, 2010, pp. 64-68.

[15] Chen L., Chen S. C.: Distance-based sparse associative memory neural network algorithm for pattern recognition. Neural Processing Letters, 2006, 24, pp. 67-80.

[16] Chen S. C., Chen L., Zhou Z. H.: A unified SWSI-KAMs framework and performance evaluation on face recognition, Neurocomputing, 2005, 68, pp. 54-69.

[17] Bishop C. M.: Pattern recognition and machine learning. Springer Science+Business Media LLC, Philadelphia, USA, 2006. (http://research.microsoft.com/ cmbishop/PRML).

[18] Liu Y., Zhang D. S., Lu G. J., Ma W. Y.: A survey of content-based image retrieval with high-level semantics, pattern recognition, 2007, 40, pp. 262-282.

[19] Zhang J. W., Zhan K. Ma Y. D.: Rotation and scale invariant antinoise PCNN features for content-based image retrieval. Neural Network World, 2007, 17, 2, pp. 121-132.

[20] Azimi-Sadjadi M. R., Jaime Salazar J., Srinivasan S.: An adaptable image retrieval system with relevance feedback using kernel machines and selective sampling. IEEE Transactions on Image Processing, 2009, 18, 7, pp. 1645-1659.

[21] Kulkarni A. D., Gunturu H., Datla S.: Association-based image retrieval. WSEAS Transactions on Signal Processing, 2008, 4, 4, pp. 183-189.

[22] Kulkarni A. D., Brown L. L.: Association-based image retrieval. In: Semantic Mining Technologies for Multimedia Databases. Dacheng Tao, Xu Dong, Xuelong Li (Editors). IGI Global, Hershey, PA, 2009, pp. 379-406.

[23] Proakis J. G.: Digital Communications, 4th ed., McGraw-Hill, New York, 2001. 